\title{
3D CELLULAR AUTOMATA FRACTURE MODEL FOR POROUS GRAPHITE MICROSTRUCTURES
}

\author{
Yelena Vertyagina ${ }^{1}$, and T. James Marrow ${ }^{2}$ \\ ${ }^{1}$ Postdoctoral Research Assistant, University of Oxford, UK \\ ${ }^{2}$ James Martin Professor of Energy Materials, University of Oxford, UK
}

\begin{abstract}
Nuclear graphite has a complex porous microstructure, which depends on raw materials and manufacturing process; porosity can change with radiolytic oxidation and also in the absence of oxidation with very high neutron fluences. Porosity directly affects the fracture process and the graphite tensile strength. To understand the effects of porosity on component strength and its relation to small specimen data, microstructure sensitive models are needed that can simulate the statistics of strength of porous microstructures, also addressing size and strain gradients effects such as notches. This requires multiscale models that capture the key microstructural features with sufficient fidelity, and also with sufficient computational economy to simulate component behaviour. To achieve this, an innovative technique to calculate the elastic stress distribution in a 3D porous solid under uniaxial or biaxial tension has been developed that uses cellular automata. Synthetic microstructures with arbitrary distributions of pore sizes and shapes are created that simulate realistic microstructures; a fracture algorithm simulates failure initiation and crack growth. The model calculates the tensile strength of a microstructure volume for any arbitrary failure criteria; the critical strain energy release rate is used as an example to demonstrate how porosity affects the fracture process. The presented Cellular Automata (CA) model is at least an order of magnitude more efficient than finite element methods of equivalent discretisation; CA are also scale independent and well suited for parallel computing. This would allow large volumes of representative microstructures to be simulated, with a Monte-Carlo based approach to investigate strength variability.
\end{abstract}

\section{INTRODUCTION}

Graphite is used as a moderator in the UK advanced gas-cooled nuclear reactors (AGR). The porosity of the graphite microstructure increases with radiolytic oxidation in the reactor, and increasing porosity causes the mechanical properties to degrade (Babout et al. 2005; Brocklehurst and Kelly 1993; Kelly et al. 1983). There is much experimental evidence for a relationship between the porosity of the microstructure and its strength, which may be characterised by a linear, power or exponential relationship, depending on the material and pore geometry.

Modelling techniques are usually employed to forward predict the behaviour of the microstructure in complex external conditions and study the change of the microstructural characteristics that affect the strength-porosity relationship. However, graphite has a very complex system of irregularly shaped pores (Figure 1); the pore size ranges from several nanometres to several millimetres, which makes graphite a difficult material to simulate. Pore shape approximation by spheres or ellipsoids may not necessarily represent the graphite microstructure adequately. Various attempts have been performed in the recent years to develop 3D heterogeneous models of fracture in graphite using FE-based methods (Berre et al. 2006; Saucedo-Mora and Marrow 2014; Smith et al. 2013) or lattice models (Morrison et al. 2014). The use of FE methods introduces some difficulties since it can require particular algorithms to be applied for creation of the FE-mesh around the pores of irregular shapes while enlargement of the mesh to simulate representative volumes of material leads to increasingly time consuming computations, especially in 3D. 
In this connection the development of an efficient microstructure-based model is required, which takes into account the real pore geometry in graphite.

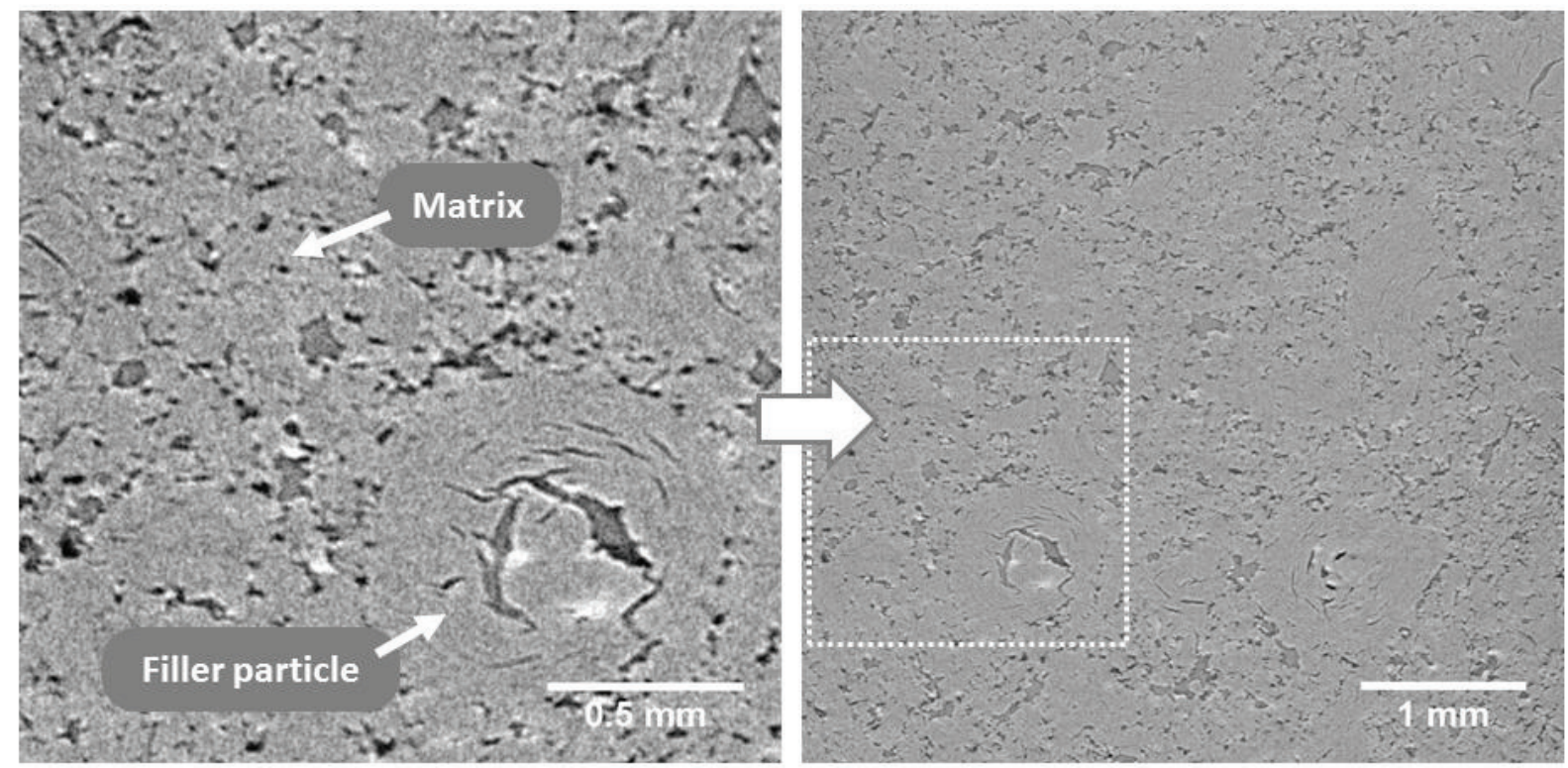

Figure 1. Computed X-ray tomography image of Gilsocarbon microstructure.

In this work, we present a novel approach for the 3D stress and fracture analysis of a microstructure with pores of arbitrary shapes. The approach is based on the Cellular Automata (Wolfram 2002) technique and applied to both synthetic microstructures and segmented tomography data of virgin Gilsocarbon graphite, which is used in the UK AGR cores. The results demonstrate the effect of pore shape on the material strength in an examination of simulated microstructures with spherical, ellipsoidal and arbitrary pores. A fracture simulation using tomography data of pore shapes and size distributions predicts different behaviour of the filler particles and surrounding matrix volumes of the heterogeneous Gilsocarbon graphite microstructure.

\section{MORPHOLOGICAL CHARACTERISATION OF POROUS GRAPHITE}

Characterisation of graphite morphological properties was performed using two tomography datasets of the virgin Gilsocarbon microstructure each at a resolution of $1.8 \mu \mathrm{m} / \mathrm{voxel}$. These data were obtained in experiments EE9036 and EE8519 at the Diamond Light Source using camera module 3 (I12 beamline) and are from the same billet of graphite (GCMB grade, manufactured by UCAR). The total number of filler particles within the considered volume of $89.8 \mathrm{~mm}^{3}$ of the first tomography dataset is 112; the filler particle volume fraction is $25.2 \%$. The second tomography dataset contains 120 filler particles with a volume fraction of $14.4 \%$ within the total volume of $77.0 \mathrm{~mm}^{3}$.

A total of 20 randomly selected filler particles have been extracted from both tomography datasets, and 7 volumes of the matrix from the first dataset only (it was not possible to reliably segment matrix pores in the second). The shape of the unbroken Gilsocarbon filler particles is typically ellipsoidal, often close to a spherical shape: only the microstructures within each ellipsoid were considered. Within each volume the microstructures were segmented using an image intensity threshold to define the resolved pores. Typical pore microstructures of a filler particle and matrix are presented in Figure 2. 
a)

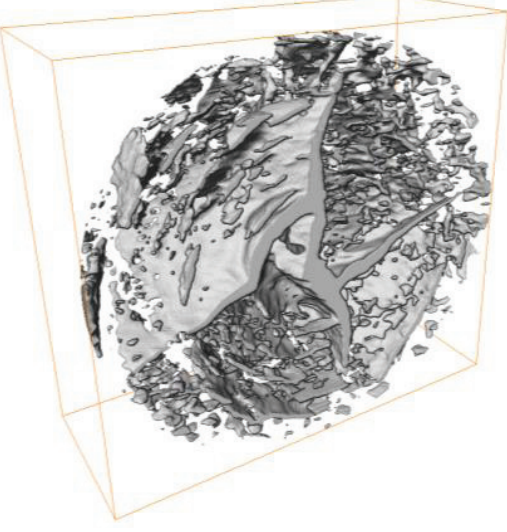

b)

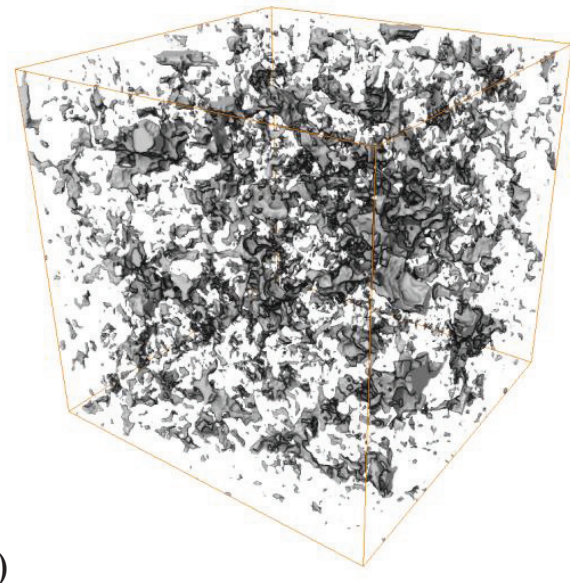

Figure 2. Typical porous microstructure of a filler particle (a) and matrix volume (b). The size of the bounding box is $0.93 \times 0.36 \times 0.79 \mathrm{~mm}$ for the filler particle and $1 \times 1 \times 1 \mathrm{~mm}$ for the matrix.

The distribution of the size of the filler particle volumes in the presented samples is shown in Figure $3 \mathrm{a}$, together with the porosity distribution in the filler particles and matrix volumes (Figure $3 \mathrm{~b}$ ). Each matrix volume has a size of $1 \mathrm{~mm}^{3}$ and the mean filler particle volume size is $0.4 \mathrm{~mm}^{3}$. The mean porosities are $12.2 \%$ for the filler and $16 \%$ for the matrix.

The measured pore size distributions in the selected volumes are presented in Figure 4. The pores vary significantly in shape and size, covering a wide range of volumes from 6 to $10^{6} \mu^{3}$; more than $90 \%$ of the total pore volume in these local regions may be spatially combined into one large pore. The most frequent pore volume is approximately $100 \mu \mathrm{m}^{3}$ for the matrix pores and $1000 \mu \mathrm{m}^{3}$ for the filler pores. It is important to note that the selection of matrix volumes excludes large matrix pores, which are important in fracture initiation (Joyce et al. 2008; Mostafavi et al. 2013b). The largest pores in the considered volume occupy of about $10^{6} \mu \mathrm{m}^{3}$ in the filler and $10^{5} \mu \mathrm{m}^{3}$ in the matrix, and the most frequent pore length is $30 \mu \mathrm{m}$ in the filler and $10 \mu \mathrm{m}$ in the matrix. The matrix pores are observed to be oriented anisotropically; this preferred orientation of pores might be explained by crystallographically oriented pores arising from flow alignment of the pitch mesophase during graphite production; the matrix data were taken from one sample.

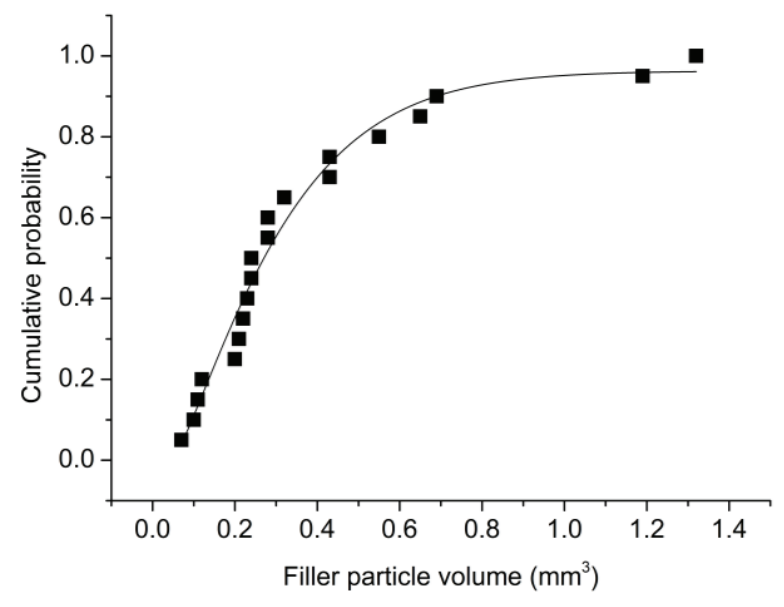

a)

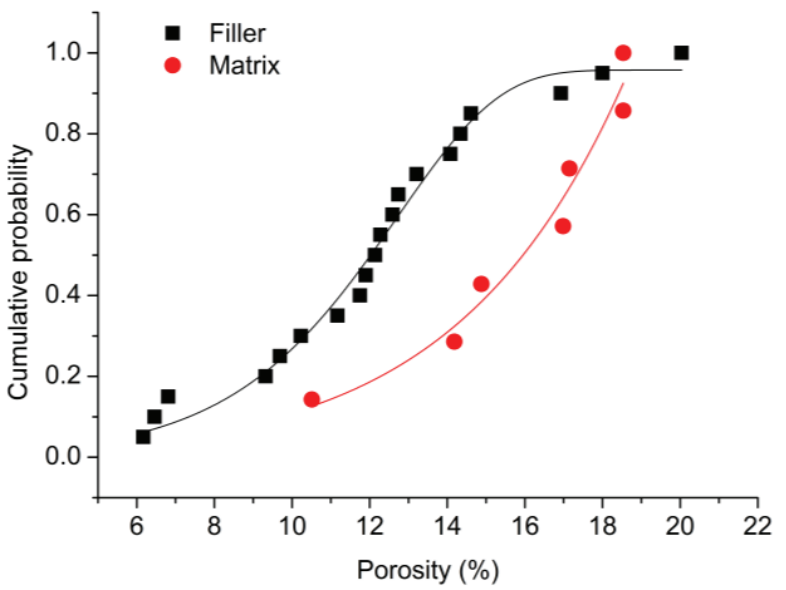

b)

Figure 3. Cumulative distributions for filler particle volumes (a) and porosities of the filler particles and matrix volumes (b). 

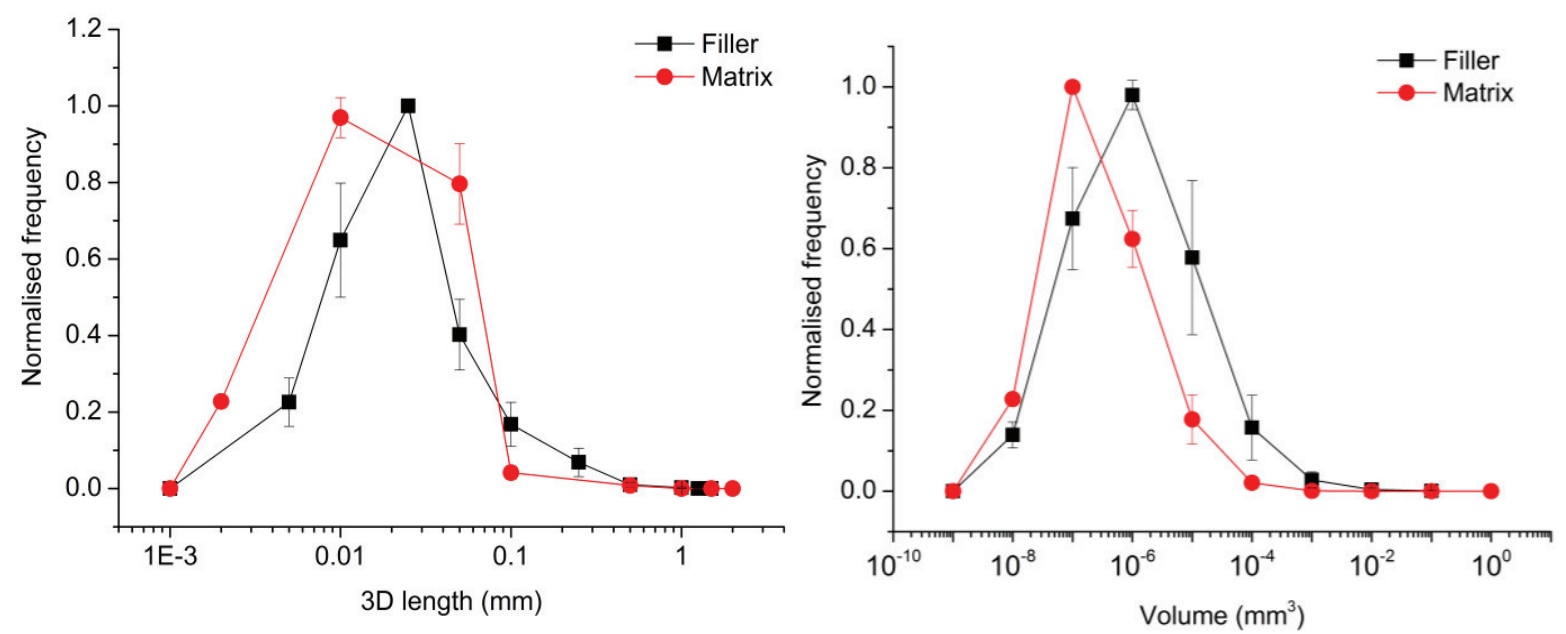

Figure 4. Pore size distributions for the filler particles and matrix.

\section{MODELLING}

\section{Cellular Automata concept for stress analysis of the microstructures with arbitrary shape pores}

The main problem of analytical approaches to stress calculation is the description of the stress field around a pore of arbitrary shape. We propose an approximation procedure based on the CA technique for the estimation of the stress concentrations in a microstructure with arbitrary pores, and consider here the case of pores subjected to uniaxial load. In our approach, a porous microstructure is considered as a CA lattice with the cells of two different states. State 1 corresponds to the cells of a solid material. These cells keep the physical parameters of the model such as the stress state and elastic energy value. The cells corresponding to the pores have state 2 ; these cells are responsible for the geometry effect during the stress calculation for the cells with state 1 .

Considering the pores as stress concentrators, the stress field created by each cell with state 2 is calculated for each cell with state 1 according to the theory of elasticity as the elastic stress around a spherical void (Timoshenko and Goodier 1951). The total stress field is obtained as the superposition of the fields produced by all cells with state 2 . The final distribution is calibrated to the analytical solution for the stress field around a spherical void. The stress concentration factor around each border cell with state 2 depends on the number of the neighbouring cells with the same state in $x, y, z$-directions to provide the ellipsoid correction (Sadowski and Sternberg 1949) along the contour of a void with an arbitrary shape. A linear elastic field has been assumed here, but the model is applicable to non-linear elastic and elastic-plastic fields with corresponding modifications.

The main advantage of the proposed technique is the possibility to calculate approximately the stress distribution in microstructures with any shapes of the pores; this computation is 10 times faster than FE simulation (considered in terms of the number of operations required for a $3 \mathrm{D}$ model with the same discretisation). This enables the iterative modelling of damage evolution that is required for fracture simulation. The simulations were done using parallel computations, carried out with the use of the Advanced Research Computing (ARC) centre of Oxford University.

\section{Fracture simulation}

A fracture algorithm for simulating damage development under a uniaxial tensile load has been developed using the CA method for stress analysis in microstructures with arbitrary shape pores. Each 
cell of the CA lattice has a local state described by the local values of the stress, strain energy and Young modulus. The cooperative behaviour of the cells leads to the global state of the system, which is characterised by the global values of the applied stress, total elastic energy and bulk Young modulus. Therefore, the feedback loop between local and global parameters may be applied to regulate the system evolution using the following steps:

- The local stresses are calculated on the basis of the applied stress value taking into account the pore geometry with the use of the proposed CA solution. This allows the estimation of the elastic energy values in each cell of the lattice.

- A fracture criterion is applied to each cell at this step. This may be any appropriate criteria for crack initiation based on the maximum stress or strain energy values, for instance. If any of the cells satisfies the criterion, then this cell is considered as damaged with the corresponding elastic energy release. Fracture may initiate in several cells simultaneously, which gives an effect of pore distribution on the fracture pattern in the microstructure.

- The total strain energy of the system is the sum of the local values assigned to each undamaged cell. Thus, the global state can be reconsidered on the basis of the new energy value to satisfy the energy balance, and therefore, the applied stress value will be recalculated. Repeat of this algorithm will lead to the fracture development at the load conditions with the constant displacement.

Young modulus is a constant parameter at the cell level; however, the bulk modulus must depend on the porosity volume fraction. Kelly's relationship (Kelly et al. 1983) for radiolytically oxidised graphite is used to provide this dependence. We do not currently consider the relationship between Young modulus and pore geometry in the simulations and assume that this is introduced by the exponential form of Kelly's relationship. A dependence of modulus on the pore geometry will be included in a future development of the model. The following mechanical properties of Gilsocarbon have been used: Young modulus $\mathrm{E}=10.85 \mathrm{GPa}$ at $20 \%$ porosity, and Poisson's ratio $v=0.21$. The critical strain energy release rate $\mathrm{G}=0.6 \mathrm{~J} / \mathrm{m}^{2}$ has been chosen as a fracture criterion on the basis of experimental data in (Bryant et al. 1964) for the surface energy of graphite in air.

\section{Simulation of the synthetic microstructures with different pore shapes and total porosity}

The sensitivity of the model to the microstructures with various pore geometries has been examined with the use of synthetic microstructures with 3 different shapes of the pores. A cellular automata lattice with $100^{3}$ cells has been used for all models. Pore shapes were defined by shape functions that define the randomly generated pore centre position and pore volume. Varying the pore sizes and rules for pore development in three dimensions in the CA lattice, we have produced microstructures with spherical, ellipsoidal and arbitrary pore shapes. The pore volume distributions are developed to approach the same target distribution (Figure 5); the final size distributions were slightly different due to pore overlapping and the effect of pore shape. The total porosity of the simulated microstructures was varied from 2 to $80 \%$. All microstructures were obtained using the same normalised distribution function. 

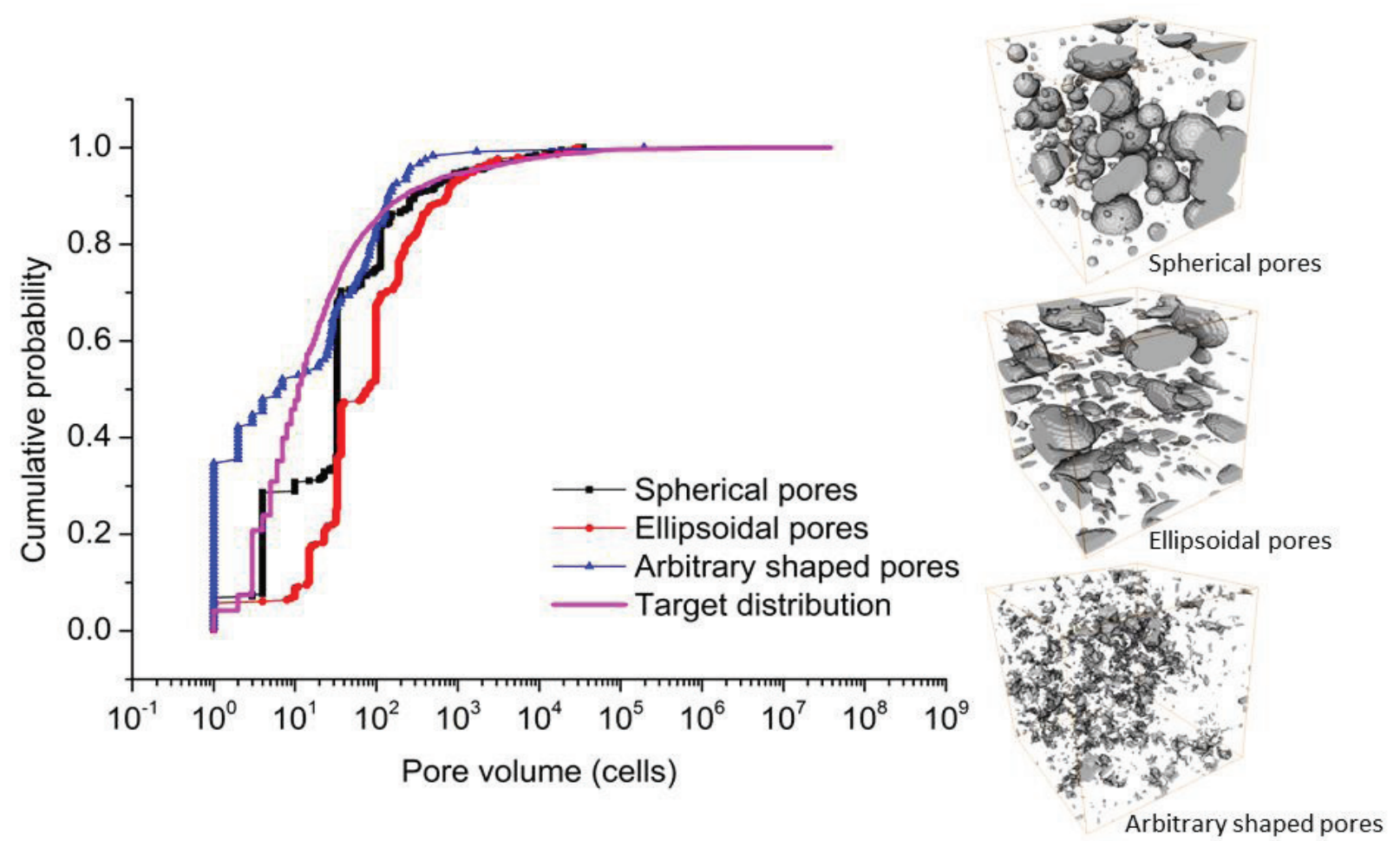

Figure 5. Example of pore size distributions for a total porosity of approximately $20 \%$ : cumulative pore size distributions for the simulated microstructures with different pore shapes.

\section{RESULTS AND DISCUSSION}

\section{Pore shape effect on the fracture process}

Fracture simulation has been performed for the synthetic microstructures with different shapes and content of pores. We examined 25 microstructures with spherical pores, 24 microstructures with ellipsoidal pores and 45 microstructures with arbitrary shaped pores within the porosity range of $2-80 \%$. The obtained dependence of the failure stress on the porosity volume fraction is presented in Figure 6; the failure stress is the peak stress sustained by the microstructure. In the majority of simulations, the peak stress was reached at the first appearance of damage, though in some cases damage occurred before the peak stress is reached. The Knudsen exponent (Knudsen 1959), also known as the DuckworthRyshkewitch relationship (Duckworth 1953; Ryshkewitch 1953), for exponential decrease in strength, has been fitted to characterise the strength behaviour in each case. Microstructures with spherical pores show the highest value of the Knudsen exponent. The microstructures with ellipsoidal pores have the lowest strength. In the region of high porosity values $(>55 \%)$ the difference between the microstructures disappears; this is because the pore structures are similar due to pore enlargement and coalescence. 


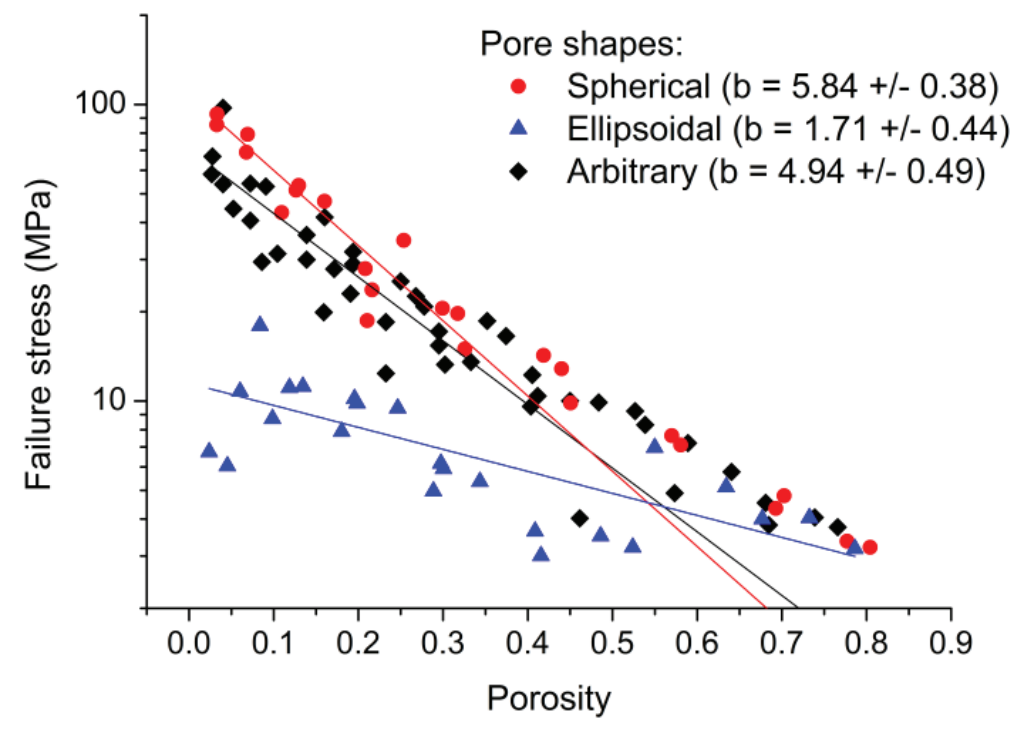

Figure 6. Dependence of the strength on the porosity for the synthetic microstructures with different shapes of the pores.

The measured Knudsen exponent is close to the available experimental values for oxidised graphite, which vary between 4.0 in (Kelly et al. 1983), 5.0 and 5.77 in (Berre et al. 2008). It is noticeable that the relationship poorly approximates the modelling results in the region of high porosity; the fracture process may have different character in microstructures with high porosity due to the reaching of the percolation threshold.

\section{Behaviour of the filler and matrix under a uniaxial tensile load}

The segmented tomography data have been used in the simulation to study possible differences in fracture behaviour in the filler and matrix volumes. Both sub-microstructures represent the same material with the only difference being the pore organisation. The data resolution has been reduced to $5 \mu \mathrm{m} / \mathrm{cell}$ in order to apply a CA lattice of $200^{3}$ cells. A total of 11 filler particles with volume sizes of no more than 1 $\mathrm{mm}^{3}$ and 7 matrix volumes each with the same size of $1 \mathrm{~mm}^{3}$ have been selected from the sets in Figure 3 . Each filler particle and matrix volume has been subjected to uniaxial tension in the $x, y$ and $z$ orthogonal directions to examine the effects of pore orientation on anisotropy.

Figure 7 presents the simulated fracture development in the filler particles and matrix. Fracture is observed to initiate within the isolated filler particle between the shell-like pores, whose proximity produces a region of high stress inside the filler particle. Generally, this process may occur in several different parts of the particle. Then, the fractured volume grows and conjunction of the pores is observed. Fracture in the matrix is observed to be more extensive due to the large number of pores and various shapes that provide favourable initiation sites.

A comparison between the failure stress distributions for the filler and matrix (Figure 8a) demonstrates that the predicted failure strengths are more tightly distributed for the matrix than the filler particles. The $50 \%$ probability failure stress is $27 \mathrm{MPa}$ for the matrix and $11 \mathrm{MPa}$ for the filler. The cumulative probabilities for the filler and matrix can be approximated by 2-parameter Weibull distributions with obtained Weibull moduli of $0.62 \pm 0.03$ for the filler and $0.44 \pm 0.07$ for the matrix. It is noticeable that the failure stress depends on the orientation of the matrix samples; this effect is less evident for the filler particles. The cumulative distributions for the failure stresses for the different tension directions are presented in Figure 8(b-d). These data show the effects of pore geometry in the modelled volumes; no relation was found between the size of the modelled volume and the predicted strength. 
a)

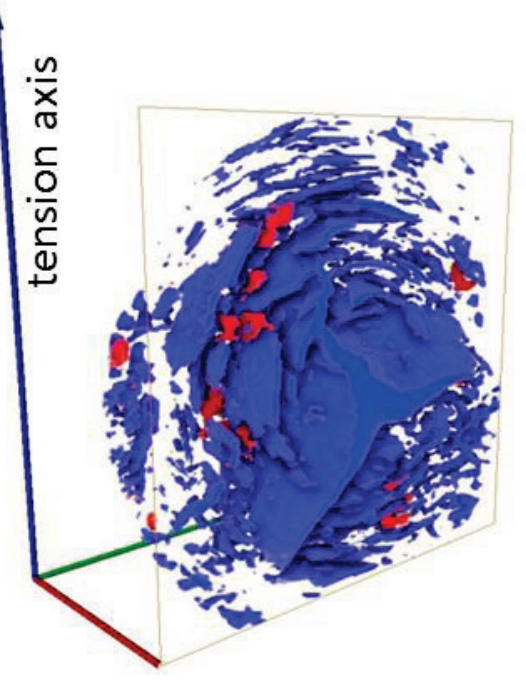

b)

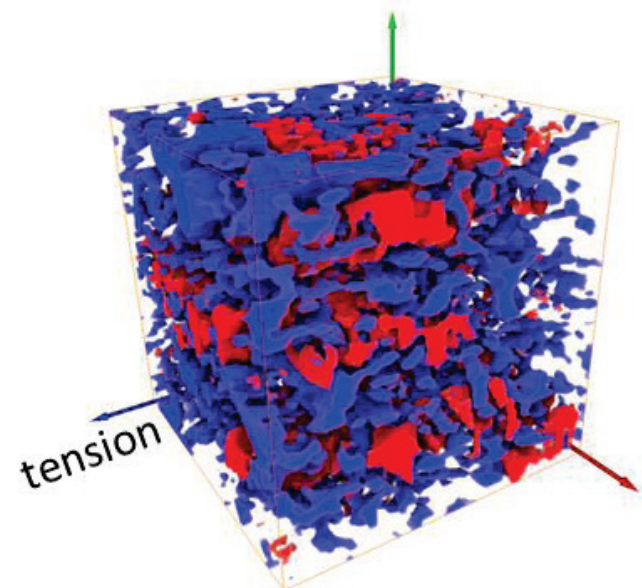

Figure 7. Fracture development simulation in the filler particle (a) and matrix (b). Pores are coloured in blue, cracked regions are red. Failure stress here is $17.7 \mathrm{MPa}$ for the filler particle and 15.1 MPa for the matrix. The damage is visualised after the peak stress.

\section{Future Perspectives}

The presented approach can be used as a part of the multi-scale fracture model, which we are developing with a combination of CA and CAFE techniques (Saucedo-Mora and Marrow 2014). The failure stress/strain values calculated at high resolution by the CA technique may then be introduced as heterogeneous material properties (together with larger pores that are not include in the CA model) within a CAFE model to simulate fracture nucleation and propagation in test specimens and components of different geometries. The general behaviour of the large scale model may then be compared with experimental observations of crack development that have been obtained for Gilsocarbon graphite, e.g. (Mostafavi et al. 2013b; Mostafavi et al. 2013a; Mostafavi et al. 2013c).

\section{CONCLUSION}

A CA approach to estimation of the three dimensional stress state in a porous microstructure has been applied to examine the fracture strengths of synthetic microstructures with different pore geometries. The simulation shows a pore shape effect on the dependence of fracture behaviour with increasing porosity; microstructures with spherical pores have better strength whilst the microstructures with ellipsoidal pores demonstrate the lowest values of the failure stress. Microstructures with arbitrary shaped pores show intermediate behaviour, which is close to available experimental data for oxidised graphite.

Morphological analysis with the use of the tomography data has been performed for the different filler particle and matrix structural features of Gilsocarbon. Different fracture behaviours of the filler and matrix have been revealed, and in the absence of large pores, which are excluded from the selection volumes, the average matrix strength obtained in the simulation is slightly higher and less variable than the filler particle strength. The matrix failure stress depends on the orientation of the sample, which indicates anisotropy at the considered length scale. 


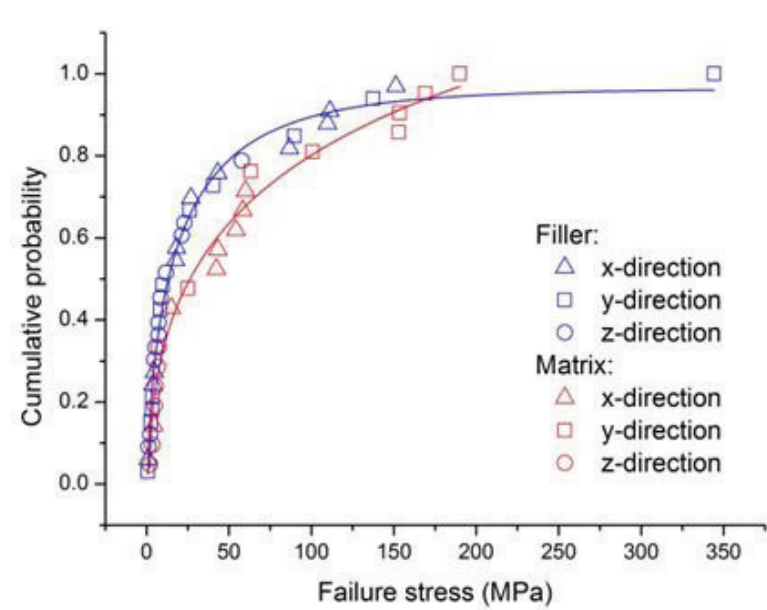

a)

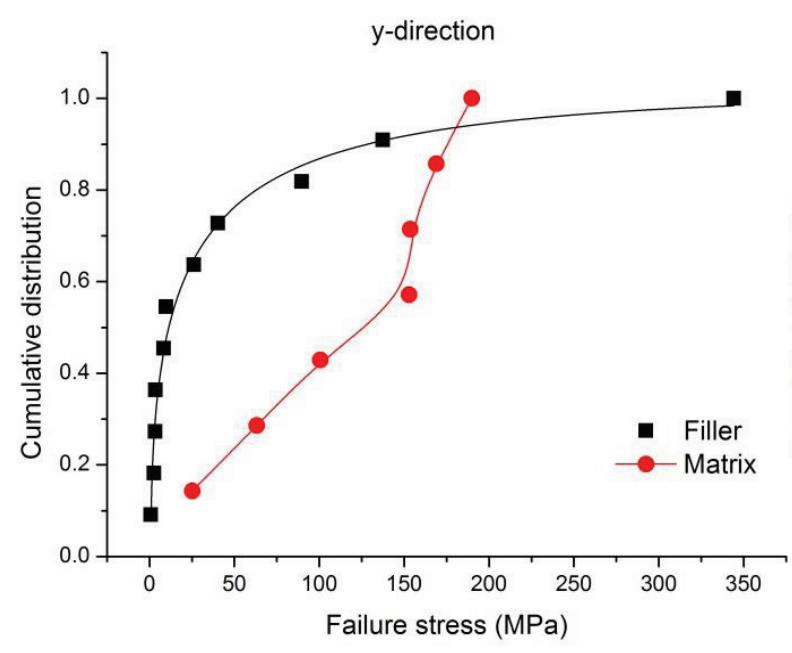

c)

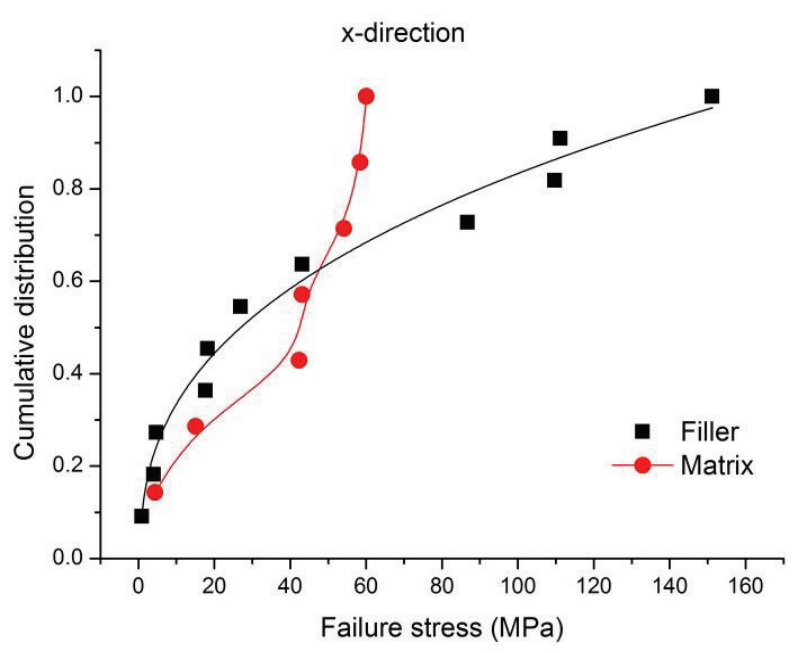

b)

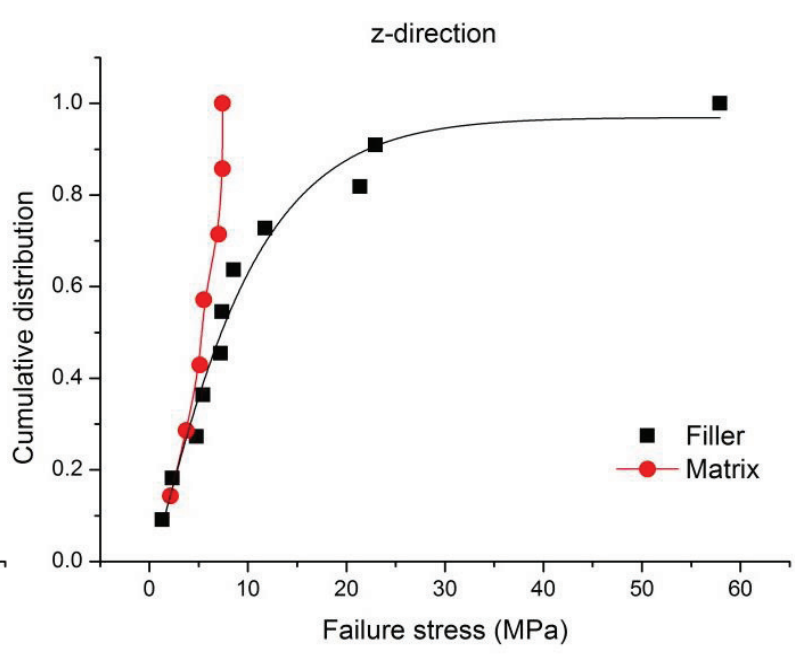

d)

Figure 8. Comparison between the failure stress distributions of the filler and matrix.

\section{ACKNOWLEDGMENTS}

T.J.M. gratefully acknowledges the support of Oxford Martin School, and Ye.V. acknowledges the support of EDF Energy Generation. The views expressed in this paper are entirely those of the authors and do not necessarily represent those EDF Energy Generation. The authors acknowledge the use of the Advanced Research Computing (ARC) in carrying out this work. Ye.V. thanks Mr M. Jordan and Dr L. Sausedo-Mora at Oxford University for fruitful discussions, and Dr P. Heard (Bristol University) for providing the target pore size distribution.

\section{REFERENCES}

Babout, L., Mummery, P. M., Marrow, T. J., Tzelepi, A., and Withers, P. J. (2005), 'The effect of thermal oxidation on polycrystalline graphite studied by X-ray tomography', Carbon, 43 (4), 765-774. 
Berre, C., Fok, S.L., Marsden, B.J., Babout, L., Hodgkins, A., Marrow, T.J., and Mummery, P.M. (2006), 'Numerical modelling of the effects of porosity changes on the mechanical properties of nuclear graphite', Journal of Nuclear Materials, 352, 1-5.

Berre, C., Fok, S.L., Mummery, P.M., Ali, J., Marsden, B.J., Marrow, T.J., and Neighbour, G.B. (2008), 'Failure analysis of the effects of porosity in thermally oxidised nuclear graphite using finite element modelling', Journal of Nuclear Materials, 381, 1-8.

Brocklehurst, J. E. and Kelly, B. T. (1993), 'Analysis of the dimensional changes and structural changes in polycrystalline graphite under fast neutron irradiation', Carbon, 31 (1), 155178.

Bryant, P.J., Gutshall, P.L., and Taylor, L.H. (1964), 'A study of mechanisms of graphite friction and wear', Wear, 7 (1), 118-126.

Duckworth, W. (1953), 'Discussion of Ryshkewitch paper', Journal of The American Ceramic Society, 36 (2), 68.

Joyce, M. R., Marrow, T. J., Mummery, P., and Marsden, B. J. (2008), 'Observation of microstructure deformation and damage in nuclear graphite', Engineering Fracture Mechanics, 75 (12), 3633-3645.

Kelly, B.T., Johnson, P.A.V., Schofield, P., Brocklehurst, J.E., and Birch, M. (1983), 'U.K.A.E.A. northern division studies of the radiolytic oxidation of graphite in carbon dioxide', Carbon, 21 (4), 441-449.

Knudsen, F.P. (1959), 'Dependence of Mechanical Strength of Brittle Polycrystalline Specimens on Porosity and Grain Size', Journal of The American Ceramic Society, 42 (8), 376-387.

Morrison, C.N., Zhang, M., Jivkov, A.P., and Yates, J.R. (2014), 'A Discrete Lattice Model of Quasi-brittle Fracture in Porous Graphite', Materials Performance and Characterization, $3(3)$.

Mostafavi, M., McDonald, S. A., Mummery, P. M., and Marrow, T. J. (2013a), 'Observation and quantification of three-dimensional crack propagation in poly-granular graphite', Engineering Fracture Mechanics, 110, 410-420.

Mostafavi, M., McDonald, S.A., Çetinel, H., Mummery, P.M., and Marrow, T.J. (2013b), 'Flexural strength and defect behaviour of polygranular graphite under different states of stress', Carbon, 59, 325-336.

Mostafavi, M., Baimpas, N., Tarleton, E., Atwood, R. C., McDonald, S. A., Korsunsky, A. M., and Marrow, T. J. (2013c), 'Three-dimensional crack observation, quantification and simulation in a quasi-brittle material', Acta Materialia, 61 (16), 6276-6289.

Ryshkewitch, E. (1953), 'Compression Strength of Porous Sintered Alumina and Zirconia', Journal of The American Ceramic Society, 36 (2), 65-68.

Sadowski, M.A. and Sternberg, E. (1949), 'Stress concentration around a triaxial ellipsoidal cavity', Journal of Applied Mechanics, 16, 149-157.

Saucedo-Mora, L. and Marrow, T.J. (2014), '3D Cellular Automata Finite Element Method with Explicit Microstructure: Modeling Quasi-brittle Fracture using Meshfree Damage Propagation', Procedia Materials Science, 3, 1143-1148.

Smith, G.E., Flewitt, P., and Schlangen, H.E.J.G. (2013), 'Multi-scale Modelling of Nuclear Reactor Core Graphite', Journal of Multiscale Modelling, 05 (01).

Timoshenko, S. and Goodier, G. (1951), Theory of elasticity (New York, Toronto, London: McGraw-Hill Book Company, Inc.).

Wolfram, Stephen (2002), A new kind of science (Wolfram Media. Inc., Champaign). 